\title{
Intellectual Disability, Coarse Face, Relative Macrocephaly, and Cerebellar Hypotrophy in
}

\section{Two Sisters}

\author{
Sérgio B. Sousa, ${ }^{1,2 *}$ Fabiana Ramos, ${ }^{1}$ Paula Garcia, ${ }^{3}$ Rui P. Pais, ${ }^{4}$ Catarina Paiva, ${ }^{5}$ \\ Philip L. Beales, ${ }^{6}$ Gudrun E. Moore, ${ }^{2}$ Jorge M. Saraiva, ${ }^{1,7}$ and Raoul C. M. Hennekam ${ }^{8}$ \\ ${ }^{1}$ Serviço de Genética Médica, Hospital Pediátrico, Centro Hospitalar e Universitário de Coimbra, Coimbra, Portugal \\ ${ }^{2}$ Clinical and Molecular Genetics Unit, UCL Institute of Child Health, London, UK \\ ${ }^{3}$ Unidade de Doenças Metabolicas, Centro de Desenvolvimento da Criança, Hospital Pediátrico, Centro Hospitalar e Universitário \\ de Coimbra, Coimbra, Portugal \\ ${ }^{4}$ Serviço de Neuroradiologia, Hospital Pediátrico, Centro Hospitalar e Universitário de Coimbra, Coimbra, Portugal \\ ${ }^{5}$ Serviço de Oftalmologia, Hospital Pediátrico, Centro Hospitalar e Universitário de Coimbra, Coimbra, Portugal \\ ${ }^{6}$ Molecular Medicine Unit, UCL Institute of Child Health, London, UK \\ ?University Clinic of Pediatrics, Faculty of Medicine, University of Coimbra, Coimbra, Portugal \\ ${ }^{8}$ Department of Pediatrics and Clinical Genetics, Academic Medical Center, University of Amsterdam, The Netherlands
}

Manuscript Received: 27 June 2013; Manuscript Accepted: 16 August 2013

We report on two Portuguese sisters with a very similar phenotype characterized by severe intellectual disability, absent speech, relative macrocephaly, coarse face, cerebellar hypotrophy, and severe ataxia. Additional common features include increased thickness of the cranial vault, delayed dental eruption, talipes equino-varus, clinodactyly, and camptodactyly of the fifth finger. The older sister has retinal dystrophy and the younger sister has short stature. Their parents are consanguineous. We suggest this condition constitutes a previously unreported autosomal recessive entity. (c) 2013 Wiley Periodicals, Inc.

Key words: intellectual disability; coarse facial features; dysmorphisms; cerebellar atrophy; cerebellar hypotrophy; ataxia; thick calvarium; retinal dystrophy; short stature

\section{INTRODUCTION}

There are a large number of conditions in which severe intellectual disability and small cerebellum occur together, and often only a few affected individuals for each entity are known [D'Arrigo et al., 2005; Poretti et al., 2008; Doherty et al., 2013]. Here we describe two sisters with this phenotypic combination who have additional features, which allow differentiation from other, known similar conditions. We propose that they have a discrete and hitherto unreported entity.

\section{CLINICAL REPORTS}

The family (Fig. 1) originates from a village in the interior central part of Portugal. Five of the siblings are unaffected, and the parents

\section{How to Cite this Article:}

Sousa SB, Ramos F, Garcia P, Pais RP, Paiva C, Beales PL, Moore GE, Saraiva JM, Hennekam RCM. 2014. Intellectual disability, coarse face, relative macrocephaly, and cerebellar hypotrophy in two sisters.

Am J Med Genet Part A 164A:10-14.

are also healthy with normal intelligence. Mother's head circumference is $57 \mathrm{~cm}$ (90th-97th centile). Parents are consanguineous (first cousins). In the same family there is another consanguineous couple (III.15 and III.16) who had three male children (IV.8, IV.10, IV.11), who all died in the neonatal period without apparent malformations and for unknown reasons. One of the daughters of this couple (IV.15) had a termination of pregnancy because of

Jorge M. Saraiva and Raoul C. M. Hennekam contributed equally. Grant sponsor: Fundação para a Ciência e Tecnologia; Grant number: SFRH/BD/46778/2008.

*Correspondence to:

Sérgio B. Sousa, M.D., Serviço de Genética Médica, Hospital Pediátrico de Coimbra, Av. Afonso Romão, 3000-602 Coimbra, Portugal.

E-mail: sbsousa@chc.min-saude.pt; sergio.sousa.09@ucl.ac.uk Article first published online in Wiley Online Library

(wileyonlinelibrary.com): 25 November 2013

DOI 10.1002/ajmg.a.36235 


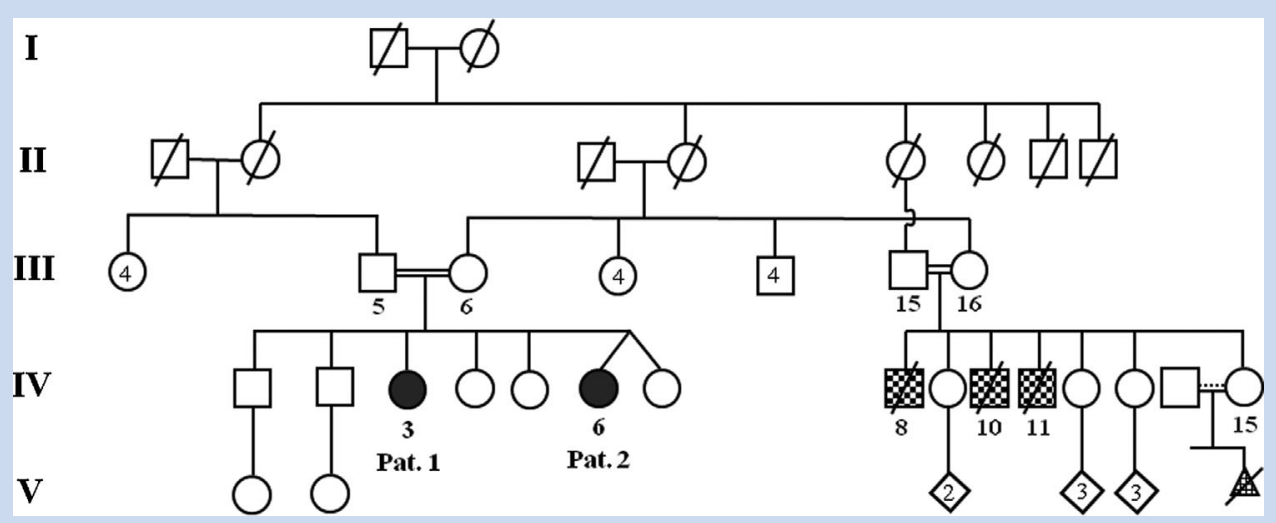

FIG. 1. Pedigree of the presented family. The dashed mating line between IV.14 and IV.15 represents possible consanguinity.

multiple fetal malformations. No additional data on these dead children could be obtained.

\section{Patient 1}

The older affected sister (IV.3) was born after an uneventful pregnancy at 40 weeks of gestation with a good start (APGAR 10 and 10 after 1 and $5 \mathrm{~min}$, respectively), and normal growth parameters (birth weight 3,600 g, length $49 \mathrm{~cm}$, OFC $35 \mathrm{~cm}$; all at the 50th centile). In the first months she was noted to be hypotonic, and at 3 months she did not smile. Gradually severe developmental delay became evident. She sat at 12 months and started to walk with help at 24 months. Walking has always been very ataxic. At 14 months she had a single seizure, without fever. She never had seizures subsequently. She did not develop any speech. Menarche was late at 15 years of age, pubertal development was otherwise normal.

We examined her at 22 years of age (Figs. 2-3). Height was $155.5 \mathrm{~cm}$ (10th centile) and OFC was $56.4 \mathrm{~cm}$ (90th-97th centile). She had severe intellectual disability, with some stereotypical movements, ataxia, apraxia, and spasticity. There were no myotatic reflexes and her plantar reflex responses were Babinski's. The extension of elbows and knees was limited. She had a significantly coarse face (Fig. 2), crowded teeth, and a very high palate. Extension of elbows and knees were limited, hands showed clinodactyly and camptodactyly of fifth fingers. There were also bilateral talipes equinovarus, the fourth toes were somewhat shortened, and the nails of the fifth toes small. Liver and spleen were not enlarged.

\section{Patient 2}

The younger affected girl (IV.6) was one of non-identical twins; her twin sister was normal. Pre-eclampsia and maternal pleural empyema resulted in a caesarean at 34 weeks. She had a good start (APGAR scores 8 and 10 at 1 and $5 \mathrm{~min}$, respectively). Weight was $2,100 \mathrm{~g}$ ( 50 th centile). At 10 days length was $42 \mathrm{~cm}$ (10th centile) and OFC $32 \mathrm{~cm}$ (50th centile). Left talipes equino-varum was noted. She had marked gastroesophageal reflux in the first months of life. Her clinical picture was very similar to that of her older affected sister: severe intellectual disability, hypotonia, delayed walking (first steps with help at 20 months), ataxia, and absent speech. She never had a seizure. The manifestations were not progressive and, to date, she has showed no deterioration. Eruption of teeth was delayed. Surgical procedures because of talipes equino-varus were performed at 4 (left foot, congenital) and 7 years (right foot, developed

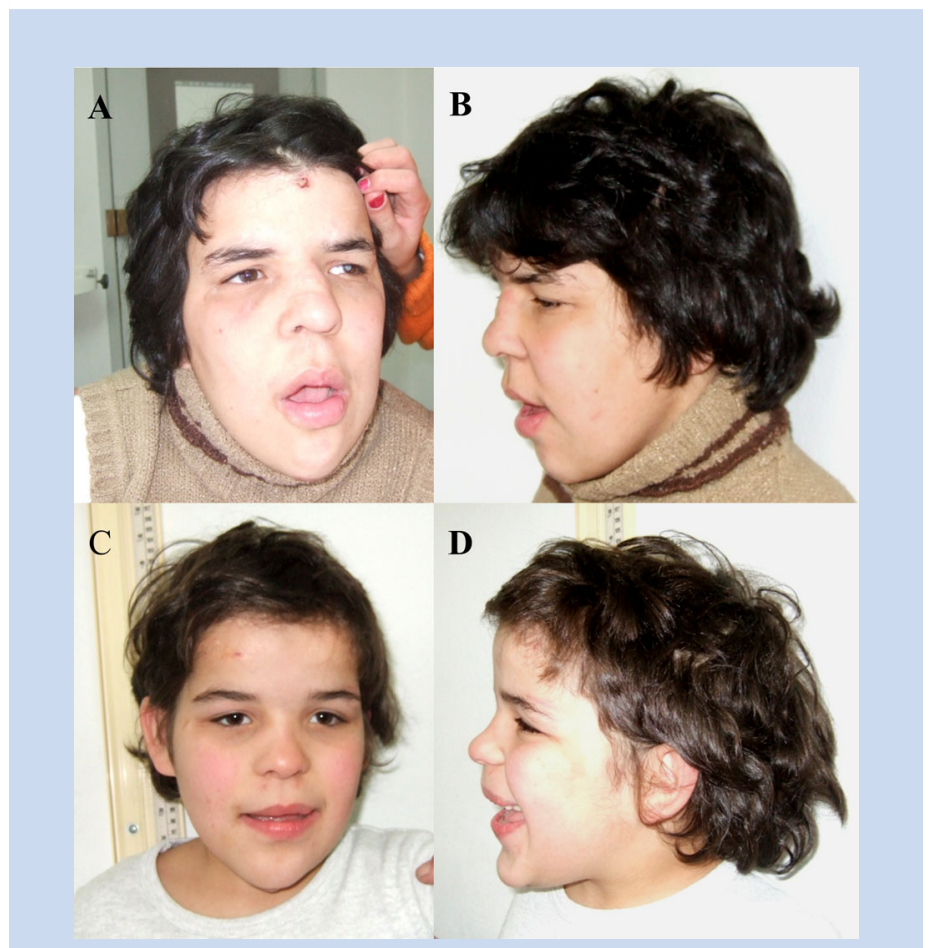

FIG. 2. Clinical pictures of Patient 1 (IV.3) at 22 years (A, B) and Patient 2 (IV.6) at 9 years and 8 months of age (C, D). Please note the coarse facial features, slightly short palpebral fissures, broad nasal base and slight underdevelopment of the alae, long and broad philtrum, thick lips, and cataract of the left eye in Patient 1. 


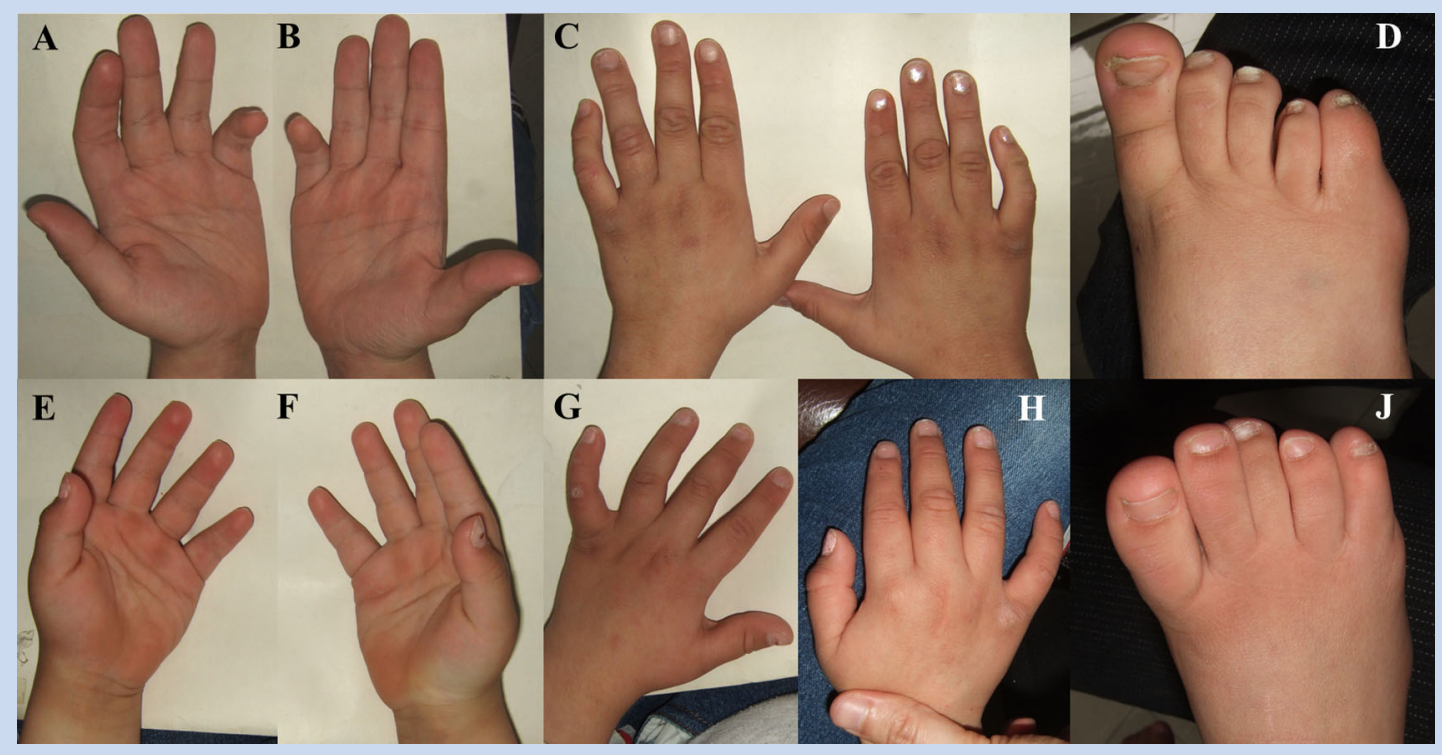

FIG. 3. Hands and feet of Patient 1 at 22 years (A-D) and Patient 2 at 9 years and 8 months of age (E-J). Please note the clinobrachydactlyly and camptodactyly of the fifth fingers, mild shortening of the fourth metatarsal in Patient 1, thick fifth toes, and slightly small fifth toenails.

postnatally). We observed her at 9.8 years (Figs. 2-3). She had short stature (height $117.5 \mathrm{~cm},-3 \mathrm{SD}$ ) and relative macrocephaly (OFC $54.6 \mathrm{~cm}, 97 \mathrm{th}$ centile). Her neurological exam was identical to that of her sister (ataxia, absent reflexes, Babinski's). She had a similar coarse face, high palate, clinobrachydactyly and slight camptodactyly of fifth fingers, and small fifth toenails (Figs. 2-3). The spasticity was more significant on the left side. At 10 years and 4 months, a developmental assessment using the revised Griffiths Mental Development Scale was performed and revealed a general quotient of 17 (corresponding to 21 months of mental age), with lower results at the Hearing and speech (quotient of 12, equivalent to 15 months) and Locomotor development (quotient of 15, equivalent to 18 months) individual scales.

\section{Additional Investigations}

In both sisters skeletal X-rays showed thickened cranial vault (Fig. 4), slightly sclerotic cranial base, poorly modelled phalanges, small fifth finger medial phalanges, and broad metacarpal and phalanges of the fifth toes (Fig. 4). In Patient 1, the fourth metatarsals were short (Figs. 3-4).

A brain MRI on Patient 1 at the age of 20 years (Fig. 5) showed significant reduced size of the cerebellum with signs predominantly suggestive of cerebellar atrophy or hypotrophy, less used but probably a more correct pathological term in this case. The cerebellum vermix and hemispheres are small but normally formed with shrunken folia and enlarged fissures. There was also some slight thinning of the bulbar brainstem and T2 and FLAIR hypersignal of periventricular white matter, diffuse, bilateral and symmetric, mostly periatrial and occipito-temporal, relatively unspecific (Fig. 5). A previous head CT scan in Patient 1 at the age of
6 years had already revealed a small cerebellum and comparing both exams, a significant progression of the cerebellar hypotrophy was not evident. A brain MRI in Patient 2 at 7 years also showed cerebellar hypotrophy (Fig. 5). A previous brain CT of Patient 2 at 3 years of age was reported as being normal but these images were not available for us to compare. In both patients, the cerebellar hypotrophy is likely to be early onset and longstanding as the posterior cranial fossa is small, the torcular herophili is low positioned and there is thickening of the occipital bone. Specially in Patient 1, but also in milder degree in Patient 2, there is thickening of the cranial vault what could be a feature of this syndrome but also an indication of some degree of cerebral atrophy (despite the normal size of ventricles and subarachnoid spaces).

An electroencephalogram in Patient 1 at 6 years showed delayed maturation, without other pathological aspects, and no abnormalities in Patient 2. Electromyography and nerve conduction studies in both patients yielded normal results as did a cardiac ultrasound in Patient 2.

Ophthalmological evaluation of Patient 1 at 22 years of age showed a unilateral cataract, which was thought to be secondary to a trauma. In the right eye, atrophy of the pigmentary retinal epithelium with visualization of choroideal vessels, peripapilar atrophy with basal crescent, osseous spicules spread at the posterior pole and macular atrophy was visible. The electroretinogram of this eye showed retinal degeneration. No eye abnormalities were identified in Patient 2 at 10 years of age. Patient 2 had conductive hearing loss that required unilateral myringotomy.

Additional studies in both sisters included classical karyotype, telomeres analyses, methylation studies for Angelman syndrome, thyroid function tests, lactate, ammonia, CK, cholesterol and TG, liver function tests, plasma levels of very long chain fatty acids and 

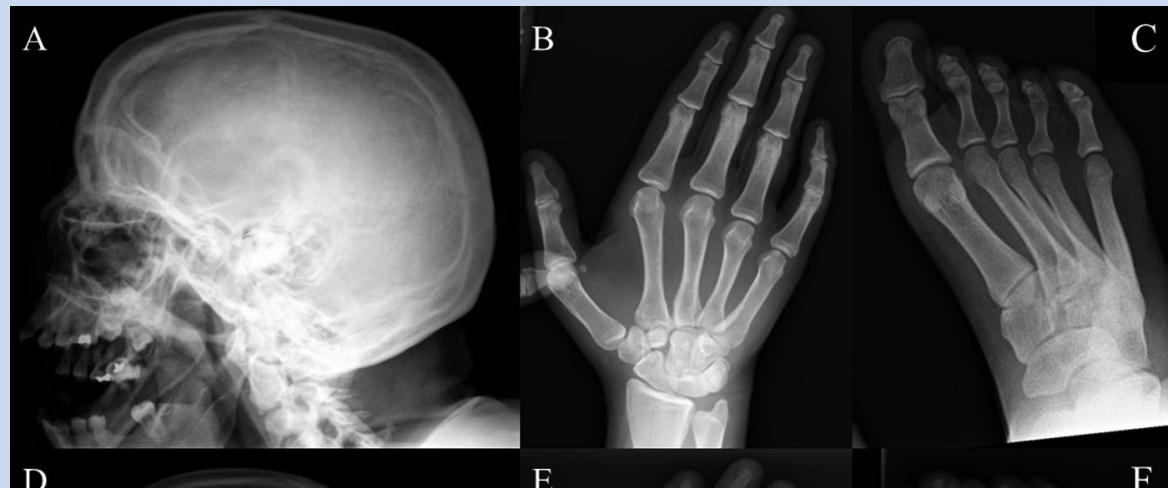

D

E

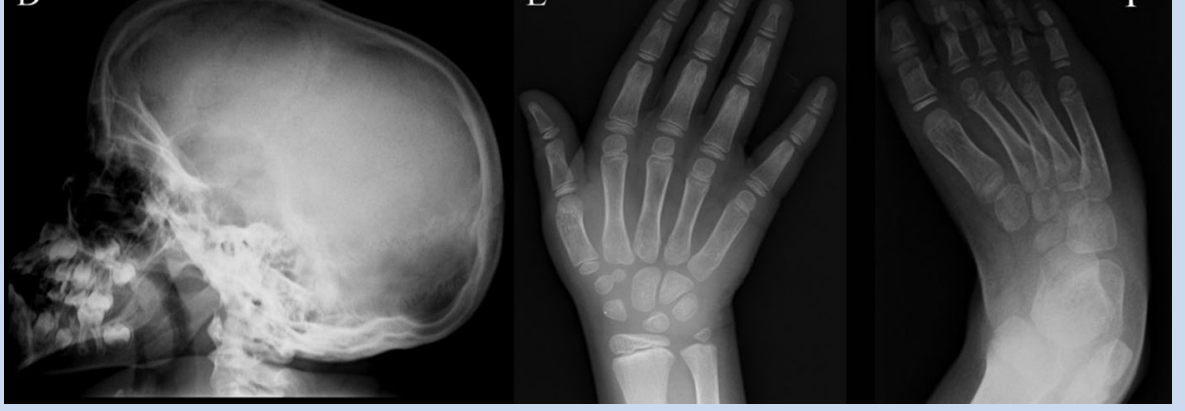

FIG. 4. Radiographs of Patient 1 at 22 years $(A-C$ ) and Patient 2 at 9 years and 8 months of age (D-E) and 6 years and 11 months $(J)$. Please note the thick cranial vault and slightly sclerotic cranial base, the poorly modeled phalanges and metacarpals, the shortening of the middle phalanges of the fifth fingers and the broad proximal and medial phalanges and small terminal phalanges of the fifth toes.

phytanic acid, lysosomal enzymes and blood film looking for vacuolated lymphocytes, urinary excretion of oligosaccharides and mucopolysaccharides, amino acids and organic acids, with all yielding normal results. Patient 1 had normal sialic acid levels in the urine and fibroblasts studies in the younger sister revealed normal total and combined sialic acid, alpha neuraminidase and beta galactosidase assays. Further studies were performed in Patient 2 included oligoarray comparative genomic hybridization (Agilent Technologies, Palo Alto, CA), prolactin and PTH plasma levels, plasma transferrin and acetylcarnitine studies, creatine/guanidinoacetic acid, sulfites and biopterin studies in urine. All results were normal. Lyonization studies in the mother at the human androgen receptor (HUMARA) locus showed random X-chromosome inactivation.

\section{DISCUSSION}

The main characteristics in the affected sisters are severe intellectual disability, absent speech, cerebellar hypotrophy, congenital hypotonia, coarse facial features, relative macrocephaly, and thick cranial vault. The hindbrain anomalies were early onset, could be progressive in childhood and in one patient also involved the brainstem. The neurological picture is dominated by the hypotonia, severe delay in reaching the motor milestones, ataxia which is especially clear in the widely base walking, apraxia, gradual increase in spasticity, and development of talipes equino-varus, areflexia and Babinski patterns of the plantar reflexes. Electrophysiological studies failed to show peripheral abnormalities, and together with the neuroradiological findings this argues in favor of a central nervous origin of the neurological phenotype. The cranial vault was thick and this was more marked in the elder patient, which may indicate it to be progressive. The coarsening of the face did not become more expressed with time.

Several conditions overlap with the presently described phenotype. The diagnostic approach in cerebellar disease in childhood is complex [D'Arrigo et al., 2005] and in particular the differentiation between cerebellar hypo/atrophy and hypoplasia can be difficult in practice [Poretti et al., 2008]. Marinesco-Sjogren syndrome (OMIM 248800) shares, as manifestations, intellectual disability, cerebellar atrophy, hypotonia, ataxia, and cataract [Ezgu et al., 2013]. However, in the present cases the facial coarseness is distinct, the cataract is present in only one of the patients, is unilateral and probably post-traumatic, and the serum CK levels are normal, making this diagnosis unlikely. Sialic acid disorders [Strehle, 2003] such as Salla disease (OMIM 604369), and atypical Refsum disorder (OMIM 614879) [Waterham and Ebberink, 2012], show clinical resemblance but metabolic studies excluded both entities. Hypoplasia of the pons and cerebellum with progressive volume loss can be characteristic of congenital disorder of glycosylation type 1a [Grünewald, 2009], but many other manifestations differ and isoelectric focussing of transferrin yielded normal results. Mutations in OPHN1 (OMIM 300486) cause an X-linked intellectual disability-small cerebellum syndrome which sometimes is associated with macrocephaly [Chabrol et al., 2005], but the overall phenotype is milder and females carriers are usually asymptomatic 




FIG. 5. Brain MRIs in Patient 1 at 20 years old (A, B, E, F, G) and of Patient 2 at 7 years of age $(C, D)$. Mid-sagittal $(A, C)$ and coronal $(B, D)$ T1-weighted sections showing small cerebella, more significant in Patient 1 with thinning of the brainstem and superior cerebellar peduncles. Please note the thin and long cerebellar folia and enlarged fissures suggestive of hypo/ atrophy; and the increased thickness of the cranial vault, especially in Patient 1 and best seen in the para-sagittal sections (E). In Patient 1, there is also a hyperintense signal of supero-posterior periventricular white matter in T2 Flair-weighted sections, apparently involving the optic radiations $(F)$.

or have mild intellectual disability. Mutations in CASK (OMIM 300749) cause an X-linked dominant intellectual disability-pontocerebellar hypoplasia syndrome with absent speech, axial hypotonia, and spasticity [Burglen et al., 2012]. Contrary to the phenotype presented here, there is typically a progressive microcephaly and no facial coarsening. Several other conditions grouped together under the diagnosis pontocerebellar hypoplasia, should also be considered in the differential diagnosis [Namavar et al., 2011; Doherty et al., 2013]. When considering the whole group the present sisters differ on several important points, such as the presence of a coarse face, the thick cranial vault, the absence of microcephaly, and the absence of epilepsy. Together these characteristics allow a clear distinction from each of the previously delineated pontocerebellar hypoplasias.

We conclude that the two sisters show likely a hitherto undescribed entity characterized by severe intellectual disability, absent speech, ataxia, cerebellar hypotrophy, relative macrocephaly and coarse facial features. The occurrence in sibs, lack of vertical transmission, and consanguinity in parents fit best with an autosomal recessive pattern of inheritance. The pedigree structure (Fig. 1), nevertheless, with two sisters-one mother of the two daughters here described and the other with three neonatal male deaths, also raises the possibility of X-linked inheritance, even in the absence of skewed X-inactivation. Further molecular studies are in progress in order to identify the etiology. We hope the present report will lead to the recognition of additional patients which will help in further clinical delineation and which may facilitate molecular analyses.

\section{ACKNOWLEDGMENTS}

We are pleased to thank the family for their cooperation. S.B.S. acknowledges a grant from Fundação para a Ciência e Tecnologia (SFRH/BD/46778/2008).

\section{REFERENCES}

Burglen L, Chantot-Bastaraud S, Garel C, Milh M, Touraine R, Zanni G, Petit F, Afenjar A, Goizet C, Barresi S, Coussement A, Ioos C, Lazaro L, Joriot S, Desguerre I, Lacombe D, des Portes V, Bertini E, Siffroi J-P, de Villemeur TB, Rodriguez D. 2012. Spectrum of pontocerebellar hypoplasia in 13 girls and boys with CASK mutations: Confirmation of a recognizable phenotype and first description of a male mosaic patient. Orphanet J Rare Dis 7:18.

Chabrol B, Girard N, N'Guyen K, Gérard A, Carlier M, Villard L, Philip N. 2005. Delineation of the clinical phenotype associated with OPHN1 mutations based on the clinical and neuropsychological evaluation of three families. Am J Med Genet Part A 138A:314-317.

D’Arrigo S, Vigano L, Bruzzone MG, Marzaroli M, Nikas I, Riva D, Pantaleoni C. 2005. Diagnostic approach to cerebellar disease in children. J Child Neurol 20:859-866.

Doherty D, Millen KJ, Barkovich AJ. 2013. Midbrain and hindbrain malformations: Advances in clinical diagnosis, imaging, and genetics. Lancet Neurol 12:381-393.

Ezgu F, Krejci P, Li S, de Sousa C, Graham J, Hansmann I, He W, Porpora K, Wand D, Wertelecki W, Schneider A, Wilcox W. 2013. Phenotypegenotype correlations in patients with Marinesco-Sjögren syndrome. Clin Genet 1-11. [Epub ahead of print]. doi: 10.1111/cge.12230

Grünewald S. 2009. The clinical spectrum of phosphomannomutase 2 deficiency (CDG-Ia). Biochim Biophys Acta 1792:827-834.

Namavar Y, Barth PG, Poll-The BT, Baas F. 2011. Classification, diagnosis and potential mechanisms in pontocerebellar hypoplasia. Orphanet Rare Dis 6:50.

Poretti A, Wolf NI, Boltshauser E. 2008. Differential diagnosis of cerebellar atrophy in childhood. Eur J Paediatr Neurol 12:155-167.

Strehle EM. 2003. Sialic acid storage disease and related disorders. Genet Test 7:113-121.

Waterham HR, Ebberink MS. 2012. Genetics and molecular basis of human peroxisome biogenesis disorders. Biochim Biophys Acta 1822: 1430-1441. 